\title{
Wiki Editors' Acceptance of Additional Guidance on Talk Pages — Extended Abstract
}

\author{
Sven Heimbuch, Daniel Bodemer \\ Media-Based Knowledge Construction \\ University of Duisburg-Essen \\ Lotharstr. 65, 47057 Duisburg, Germany
}

\begin{abstract}
One purpose of Wikis like Wikipedia is to collaboratively generate content. During the creation processes controversies between multiple authors might emerge that are further discussed on a Wiki article's so-called talk page. Research has shown that controversies grounded on opposing points of view and contradictory evidence can be fruitful to trigger individual elaboration processes. But it has also been shown that many Wikis are not necessarily suited to identify relevant discussion contents and thus need additional support to guide users. In an experimental study $(N=181)$ we implemented on a Wiki talk page (1) visual controversy status highlights as implicit guidance and (2) a collaboration script directing users towards discussions as explicit guidance. We analyzed their user adoption and influence on Wiki activities. Our results show that both experimental additions affect user behaviors and that in particular the visual controversy status highlights are positively adopted by users.
\end{abstract}

\section{Introduction}

Wikipedia and Wikis in general are widely used for collaboratively creating encyclopedia-like articles. These sociotechnical environments offer opportunities to discuss any article's contents on corresponding talk pages. Essential to the co-creation of user-generated content are processes of internalizing and externalizing knowledge from an individual into the Wiki or vice versa (Cress and Kimmerle 2008).

Within these processes lie potentials for emerging controversies between collaborators that are grounded on different opinions or contradictory knowledge. These controversies can induce socio-cognitive conflicts that trigger individual equilibration and elaboration processes which can be beneficial for learning (Mugny and Doise 1978).

Despite the wide acceptance of Wikis in academia, their effectiveness and efficiency for collaborative learning activities are inconclusive due to ambiguous results. Research on Wikis has proposed additional scaffolding measures to be incorporated in Wikis to improve the overall quality of writing and coordination processes (Papadopoulos, Demetriadis, and Weinberger 2013; Wichmann and Rummel 2013).

Copyright (c) 2016, Association for the Advancement of Artificial Intelligence (www.aaai.org). All rights reserved.
In our research, we successfully evaluated two types of scaffolds focusing on potential benefits for talk pages: (1) visual representations as implicit guidance (Heimbuch and Bodemer 2014), (2) collaboration scripts as explicit guidance (Heimbuch and Bodemer 2015; Heimbuch, Uhde, and Bodemer 2014). The former is meant as a minimal invasive modification to make controversial discussions and their concurrent state more salient by adding visual highlights. Whereas the latter is proposed to be an alternative to Wikipedia's Be Bold principle and aims at fostering deeper elaboration processes by encouraging discussions prior to the externalization of knowledge into a Wiki.

Both types of proposed scaffolds for Wikis aim at focusing readers and editors towards relevant contents on discussion pages and by extension provide guidance for content creation and knowledge construction processes. The present study compares participants' adoption of both types of guidance support in two experimental Wiki-based learning environments and analyzes the individual effects on Wiki-related activities.

\section{Methods}

A two-group experimental study has been conducted with $N$ $=181$ students ( 136 females; 45 males) with an average age of $M=20.59(S D=2.59)$, mainly recruited from the Applied and Cognitive Media Science (92.80\%). The majority of participants rated themselves as being medium to highly experienced $(67.96 \%)$ with Wikis and Wikipedia. Figure 1 depicts the study's Wiki modifications for the two experimental groups.

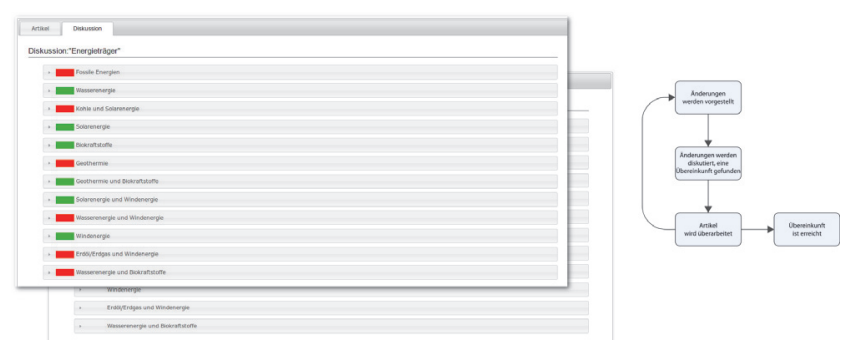

Figure 1: Controversy highlights as implicit guidance are represented on the upper left. Collaboration script representation as explicit guidance is represented on the right. 
The implicit guidance group had been working in a Wiki with additional visual representations adjacent to talk page discussion titles. The color of visualizations reflected the concurrent state of a controversial discussion: green for a consensus between discussants, red for an unresolved controversy.

The explicit guidance group was introduced to the $D D R$ collaboration script that suggested to (1) discuss proposed changes, (2) deliberate and find a temporary consensus and (3) revise the corresponding article content.

In both groups students had to read a Wiki article on energy sources and the corresponding talk page that contained twelve discussion threads, with six resolved and six unresolved controversies between discussants. A controversial discussion took place when two or more discussants were presenting evidence for contradictory points of view on a topic. Resolution was achieved when discussants reached a consensus, otherwise the controversy remained unresolved. The students' main task was to actively participate in the Wiki by commenting on self-selected discussions and edit the original article. After completion of the article revision and talk page participation, students were surveyed on cognitive variables and their experience with the modified Wikis.

In order to measure the acceptance of our guidance implementations the User Experience Questionnaire (UEQ) has been used (Laugwitz, Held, and Schrepp 2008). Further we collected log data on Wiki activities (e.g. topic selections, reading and commenting times) and assessed learning about the Wiki's topic with a multiple choice test.

\section{Results and Discussion}

The following results are still preliminary and will be described in brief and more qualitatively. Overall, participants in the implicit guidance group rated the controversy status highlights in five out six $U E Q$ dimensions higher than the explicitly guided group rated the $D D R$ script. Detailed comparisons of the six $U E Q$ dimensions between the experimental groups are shown in figure 2.

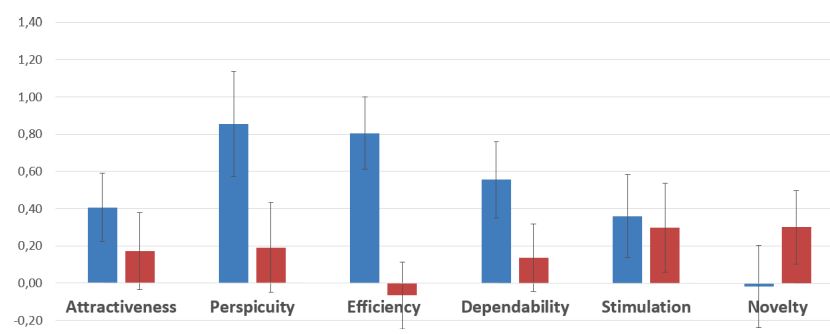

Figure 2: Mean ratings on the $U E Q$ dimensions for implicit (blue) and explicit guidance (red) implementations.

The only dimension were the explicitly guided group rated their Wiki modification significantly higher was the aspect of novelty $(t(179)=-2.11, p=.037, d=0.31)$. This could be because most students were not familiar with this kind of structured collaboration via stepped scripts.

The largest and most significant difference between both group was on the ratings of efficiency $(t(179)=6.44, p<$ $.001, d=0.96)$. Students in the implicitly guided group rated their controversy status visualization as rather positive in terms of efficiency whereas the explicitly guided experimental group gave even negative scores on the efficiency scale for the collaboration script.

Regarding the effects of modified Wikis on activities, previous studies on both guidance measures have shown that they trigger the intended behaviors, such as a focused topic selection or an a priori discussion of article changes (Heimbuch and Bodemer 2015).

The present study will be analyzed and discussed in more detail on the resulting Wiki contents and individual learning about the Wiki topics.

\section{References}

Cress, U., and Kimmerle, J. 2008. A systemic and cognitive view on collaborative knowledge building with wikis. International Journal of Computer-Supported Collaborative Learning 3(2):105-122.

Heimbuch, S., and Bodemer, D. 2014. Supporting Awareness of Content-related Controversies in a Wiki-based Learning Environment. In Proceedings of The International Symposium on Open Collaboration, OpenSym '14, 30:1-4. New York, NY, USA: ACM.

Heimbuch, S., and Bodemer, D. 2015. Let's Talk about Talks: Supporting Knowledge Exchange Processes on Wiki Discussion Pages. In International AAAI Conference on Weblogs and Social Media Ninth International AAAI Conference on Web and Social Media, 56-61. Palo Alto, California, USA: AAAI Press.

Heimbuch, S.; Uhde, K.; and Bodemer, D. 2014. "A new Wiki way?" - An experimental study of collaborative knowledge building scripts. In Liu, C.-C. et al., ed., Proceedings of the 22nd International Conference on Computers in Education., volume WIPP, 10-12. Nara, Japan: Asia-Pacific Society for Computers in Education.

Laugwitz, B.; Held, T.; and Schrepp, M. 2008. Construction and evaluation of a user experience questionnaire. In Holzinger, A., ed., HCI and Usability for Education and Work, 63-76. Graz, Austria: Springer Berlin Heidelberg.

Mugny, G., and Doise, W. 1978. Socio-cognitive conflict and structure of individual and collective performances. European Journal of Social Psychology 8(2):181-192.

Papadopoulos, P.; Demetriadis, S.; and Weinberger, A. 2013. Make it explicit!: Improving collaboration through increase of script coercion. Journal of Computer Assisted Learning 29(4):383-398.

Wichmann, A., and Rummel, N. 2013. Improving revision in wiki-based writing: Coordination pays off. Computers \& Education 62:262-270. 